\title{
APROXIMACIÓN AL DISEÑO DE UN MODELO DE VALORACIÓN DE LA RESILIENCIA EN DESTINOS TURÍSTICOS
}

\author{
Cecilia Gutiérrez Vega \\ Académica Instituto de Turismo \\ Universidad Austral de Chile \\ cgutierrezvega@gmail.com
}

\section{Resumen}

En los últimos años se han diseñado diferentes modelos de indicadores para medir la sostenibilidad de los destinos de turismo. No obstante, han quedado relegados, en estos trabajos, dos aspectos importantes: el escaso protagonismo concedido a la población local como agente relevante en el proceso de gestión turística y la excesiva importancia adjudicada a los indicadores de carácter objetivo. El papel de los componentes subjetivos cobra especial importancia en momentos de crisis y desastres naturales que afectan al sector turístico (OMT, 2005).

Apuntando a esas debilidades de los modelos existentes, el presente documento da a conocer avances en el diseño, a través del concepto de "resiliencia", definida como la capacidad de los sistemas turísticos de volver al estado de equilibrio después de haber sufrido una alteración que les afecta en su marcha diaria, de una matriz que releve de mejor forma el rol de las comunidades locales e introduzca percepciones subjetivas en la valoración de factores claves de la sostenibilidad de los destinos.

Las preguntas aquí son, por tanto: ¿Qué tipo de operaciones hacen posible su fortalecimiento? ¿Cómo se establece la articulación entre el sistema turístico y su entorno? ¿Cuáles son los factores que afectan a la vulnerabilidad de los sistemas turísticos? ¿Qué factores les hacen más resilientes? Las respuestas a estas preguntas conducen a volver la vista sobre la naturaleza de los sistemas abiertos y a indagar sobre sus más recientes estudios vinculados a la capacidad de recuperación en este tipo de sistemas turísticos.

Palabras-clave: resiliencia, desastres naturales, sistema turístico, destinos turísticos. 


\title{
APPROACH TO THE DESIGN OF A MODEL TO ASSESS THE RESILIENCE IN TOURIST DESTINATIONS
}

\author{
Cecilia Gutiérrez Vega \\ Académica Instituto de Turismo \\ Universidad Austral de Chile \\ cgutierrezvega@gmail.com
}

\begin{abstract}
In recent years, different models of indicators have been designed to measure the sustainability of tourism destinations. However, two important aspects have been relegated in these works: the limited role given to the local population as a relevant agent in the tourism management process and the excessive importance attached to objective indicators. The role of the subjective components is particularly important in times of crisis and natural disasters that affect the tourism sector (UNWTO, 2005).

Pointing to these weaknesses of existing models, this document presents advances in design, of a matrix that relieves in a better way the role of local communities and introduces subjective perceptions in the evaluation of key factors of the sustainability of the destinations.through. These matrix it is focused in the concept of "resilience", defined as the ability of tourist systems to return to the state of equilibrium after having undergone an alteration that it affects them in their daily march.

The questions are, therefore: ¿What kind of operations makes possible the strengthening of resilience? ¿How is the articulation between the tourist system and its environment established? ¿What are the factors that affect the vulnerability of tourism systems? What factors make them more resilient.
\end{abstract}

Keywords: resilience, natural disasters, tourist system, tourist destinations, vulnerability 


\section{Introducción}

En los últimos años se ha realizado un trabajo importante relativo a la sostenibilidad del turismo y a la forma en que los indicadores pueden favorecer la adopción de mejores decisiones y medidas. Cada vez más, la elaboración y utilización de estos instrumentos se considera parte fundamental de la planificación y gestión general de los destinos y es un elemento integrante de las actividades de promoción de un desarrollo sostenible del sector turístico en todos los niveles (OMT, 2005).

Se han diseñado diferentes modelos de indicadores, pero han quedado relegados dos aspectos importantes, el escaso protagonismo concedido a la población local como agente importante en el proceso de gestión turística y la excesiva importancia adjudicada a los indicadores de carácter objetivo. El papel tan importante que los componentes subjetivos tienen en la satisfacción de los clientes internos (población local) y externos (turistas) $y$, por tanto, en la sostenibilidad de los destinos turísticos, toma una gran importancia en momentos de crisis económica y de desastres naturales que afectan a los destinos.

Estos aspectos subjetivos son recogidos en el estudio propuesto por Sancho et. al. (2007), que además identifica indicadores según el grado de desarrollo de cada destino. Pero la situación actual de los sistemas turísticos, sometidos a condiciones de riesgo, vulnerabilidad, desastres naturales, cambio climático e inestabilidad económica, marcan un nuevo reto al que los sistemas estáticos no pueden dar respuesta. Los sistemas turísticos, por el contrario, son dinámicos y deben adaptarse a los cambios que se generan en el entorno $\mathrm{y}$ en su propio interior, absorber las fluctuaciones y recuperar un nuevo equilibrio del sistema. Esto es lo que intenta abortdar el concepto y el marco de análisis de la resiliencia.

Se define resiliencia como la capacidad de los sistemas vivos y en particular de las personas y los grupos humanos de volver a un estado de equilibrio después de haber sufrido un desastre natural, una crisis o una alteración de cualquier tipo.

En el sistema humano la resiliencia está muy próxima a la habilidad y las capacidades que tienen individuos y grupos sociales de de adaptarse acondiciones cambiantes. Habilidades para resurgir de las crisis, salir reforzados y aprender de ellas.

La resiliencia y su rol en los sistemas turísticos constituyen la base de este artículo, que pretende aportar nuevas directrices en la investigación del turismo en épocas de cambio, alteraciones, desastres y crisis. 
No existe total claridad respecto a quien y donde se comenzó a utilizar el término resiliencia (Kalawski \& Haz, 2003). Algunos la vinculan a las ciencias humanas, otros a la biología o a la física (Kotliarenc et al, 1999) y ha llegado a ser de uso generalizado en el campo de la psicología (Scoville 1942) y la psiquiatría. No obstante, lo que es realmente importante es que ha sido y, continúa siendo, ampliamente aplicado a numerosas situaciones y contextos para explicar la capacidad de resistir y reaccionar ante situaciones y hechos adversos.

Luthar y otros (2000), señalan que, en el área de la intervención psicosocial, la resiliencia intenta promover procesos que involucren al individuo y su ambiente social, ayudándolo a superar adversidades (riesgos), adaptarse a la sociedad y tener una mejor calidad de vida.

De acuerdo con varios expertos en resiliencia (Masten, 1999; Luthar y otros, 2000; Luthar y Cushing, 1999; Kaplan, 1999) existen dos generaciones de investigadores que han trabajado esta temática. La primera, que inicia a principios de los setenta, busca identificar los factores de riego y de resiliencia que influyen en el desarrollo de niños que se adaptan positivamente a su entorno social a pesar de vivir en condiciones de adversidad. La mayoría de los investigadores de esta generación podrían adscribirse al modelo triádico de resiliencia, que consiste en organizar los factores de resiliencia y riesgo en tres grupos diferentes: los atributos individuales, los aspectos de las familias y las características de los ambientes sociales en que los individuos se hallan inmersos.

Existe otro grupo de investigadores de la segunda generación que podrían adscribirse al modelo ecológico-transaccional de resiliencia, que tiene sus fundamentos en el modelo ecológico de Bronfenbrenner (1981). Lo medular de este modelo es que el individuo se encuentra inmerso en un ambiente determinado por diversos niveles que interactúan entre sí, ejerciendo una influencia directa en su desarrollo humano, dichos niveles son: el individual, el familiar, el comunitario y vinculado a los servicios sociales, y el cultural y vinculado a los valores sociales.

El desafío de esta segunda generación es poder identificar los procesos que están en la base de la adaptación y de la resiliencia, lo que permitiría avanzar en la teoría de la resiliencia, además de permitir la definición y diseño de estrategias que permitan promover la resiliencia y por ende la calidad de vida de individuos y grupos. 
Para identificar resiliencia, es necesario que exista una adaptación positiva, que significa que un individuo o grupo ha alcanzado expectativas sociales asociadas a su etapa de desarrollo, o cuando no ha habido signos de desajuste social. En ambos casos cuando la adaptación positiva ocurre, a pesar de la exposición a la adversidad, los desastres o las crisis, se considera una adaptación resiliente.

Con el correr de los años, el término resiliencia empieza a utilizarse en diversos ámbitos, disciplinas y campos de actuación. Uno de ellos es el turismo, donde se comprende como la capacidad de los sistemas turísticos para recuperar los equilibrios o para absorber crisis, desastres naturales o fluctuaciones internas o externas, a partir $\mathrm{y}$ teniendo en cuenta fundamentalmente, sus habilidades autoorganizativas. Estas propiedades de resistir fluctuaciones y adversidades externas y autoorganizarse exitosamente para provocar un nuevo estado de equilibrio manteniendo la esencia de la comunidad y el sistema social, dependen, significativamente, de la estructura y disposición funcional de los sistemas, así como de sus capacidades iniciales, y sus modelos de gobernanza, entre otras variables (Sancho y Vélez, 2009).

Son precisamente las nuevas capacidades que desarrollan los miembros de la comunidad -cómo emprender y dirigir negocios prósperos, cómo impulsar y sostener redes de colaboración y proyectos comunitarios y cómo crear instituciones funcionales e influyentes - las que dan lugar a una mayor resiliencia (Sancho y Vélez, 2009).

A partir los años setenta y ochenta del siglo XX, tras veinte años de operar un turismo masificado en el ámbito internacional, la evolución de las prácticas turísticas ha generado un conjunto de efectos negativos, tanto económicos como sociales, políticos y culturales que transformaron y degradaron social y ambientalmente los destinos, en especial de regiones del mundo sometidas al modelo de explotación intensiva del turismo, efectos de los que hasta entonces no se tenía conocimiento y que era necesario entender a través de modelos conceptuales que explicaran la red de relaciones que se entreteje en torno a la actividad turística. De este modo se han desarrollado modelos explicativos y matrices para establecer las relaciones y articulaciones entre el turismo y todos los subsistemas de su entorno (Sancho y Vélez, 2009). 
Dos ejemplos de esta nueva concepción la ofrecen las propuestas de Gunn (2002) y Ascanio (en Schlüter, 2000). En el caso de Ascanio, el sistema turístico se articula por dos ambientes internos: el físico-empresarial (empresas, paisajes, etc.) y el comunal o humano (comunidad receptora y visitantes), ambos interrelacionados por el carácter de servicio del turismo. Este modelo hace énfasis en el papel mediador de los servicios, así como en las relaciones de comunicación entre la comunidad receptora y los visitantes, y entre los recursos y las empresas. Fuera del sistema, en el ámbito externo, se encuentran los aspectos que lo influyen: lo político, lo económico, lo psicosocial, lo tecnológico y lo sociocultural.

Gunn, por su parte, ofrece un modelo más contemporáneo sobre los componentes del sistema turístico como base conceptual para la planificación turística, concibiendo a la demanda y la oferta como las dos fuerzas principales en la conducción de dicho sistema, a la que se une: financiamientos, recursos culturales, recursos naturales, políticas gubernamentales, competencia, comunidad, emprendedores y aspectos laborales.

En general, los modelos mencionados tendieron a una generalización sobre la explicación de las relaciones entre el sistema y el entorno. Las aportaciones que hicieron representan sin duda un avance en la aplicación de la teoría de sistemas al análisis de lo turístico, particularmente al permitir estudiar las interrelaciones entre lo natural y lo social. Es el enfoque de sistemas abiertos el que sirvió de base para la construcción teóricometodológica de los modelos elaborados, particularmente el de feed-back positivo, puesto que permitió conocer los cambios que se generan en el sistema a partir de aceptar que son los estímulos del entorno, los principales generadores del cambio. (Sancho y Vélez, 2009).

No obstante, los modelos teóricos construidos para explicarlo se muestran débiles al clarificar dos aspectos: la lógica concreta de su funcionamiento, y la distinción entre los componentes que le son propios y los que corresponden a su entorno.

Por otra parte, los modelos mencionados anteriormente, empiezan a ser cuestionados al debatirse el punto de la diferenciación entre lo que corresponde propiamente al sistema turístico y lo que corresponde a su entorno, identificándose algunas limitaciones teóricas que apuntan directamente a la concepción de los sistemas abiertos:

¿Cómo se constituye un sistema turístico? ¿Qué tipo de operación hace posible su permanencia? ¿Cómo se establece la diferencia entre el sistema turístico y su entorno? ¿Cómo evoluciona este sistema y mantiene esta diferencia? (Sancho y Vélez, 2009).

De manera de incorporar elementos de diversos modelos planteados y comprender la interrelaciónqueexisteentreellos, seplanteaelsiguienteesquemaderelaciones(verFigura1). 
Figura $\mathrm{N}^{\circ}$ 1: Relaciones de los componentes claves del sistema turístico.

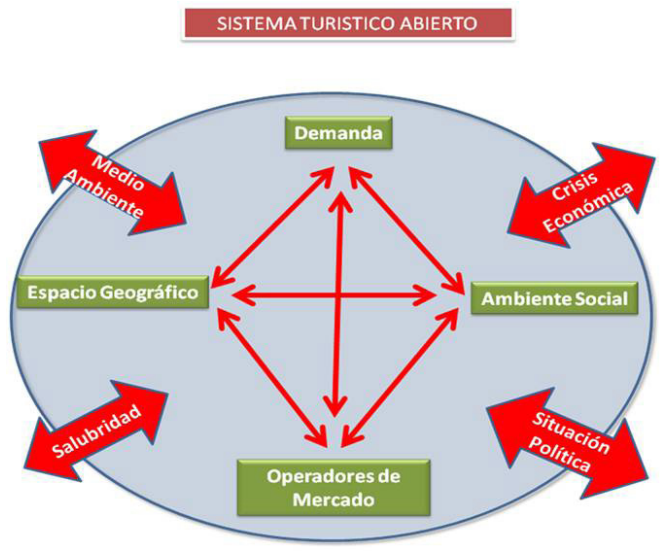

En un sistema turístico abierto como el planteado, se pueden encontrar factores endógenos y exógenos. Los cuatro principales elementos endógenos que lo conforman son:

- Demanda. Corresponde a los visitantes y turistas que arriban al destino.

- Ambiente Social. Que incluye a residentes, agentes locales vinculados al turismo, representantes de las administraciones, entre otros

- $\quad$ Operadores del Mercado Turístico. Considerando a tour operadores, agencias de viaje, hoteles y establecimientos de restauración, compañías de transporte, empresas de oferta complementaria y turismo activo, etc.

- Espacio Geográfico.Incluyendolosrecursosylosatractivosturísticos, loselementos de infraestructuray servicios públicos generales y los de infraestructuray servicios turísticos.

Existen también factores exógenos que, si bien no son propiamente del sector, afectan al sistema turístico. Entreestos se encuentran variables políticas, económicas, socio-culturales, de seguridad, ambientales, etc. En situaciones inesperadas como desastres naturales, el medioambiente ejerce su función de factor exógeno al sistema. No obstante, algunos de estos factores externos se tornan endógenos dependiendo de la relación que se genere exista con los demás elementos internos. Tal es el caso, por ejemplo, del impacto que tienen sobre el medio ambiente diversos proyectos, situaciones o eventos, que se ven afectados por los posibles usuarios del turismo, por lo que pasan a ser elementos endógenos del sistema.

Como se observa en la figura 1 , los diversos elementos que conforman un sistema turístico abierto se relacionan entre sí de una manera dinámica, entrelazándose unos con otros, ejerciendo mayor o menos presión o injerencia entre sí. 
Para poder actuar ante los riesgos, adversidades, crisis y desastres que pueden afectar al sistema turístico, es necesario ser capaces de identificar primeramente los factores claves intervinientes y desde el conocimiento de las amenazas que interfieren en el entorno ir concretando las respuestas que hay que dar ante estas amenazas. De acuerdo con lo planteado por el World Economic Fourum, (2005), existe evidencia sustancial para indicar que los riesgos exógenos afectan a los negocios hoy en día mucho más que en el pasado. Esto se debe a la profunda interrelación existente entre todos los factores socioeconómicos y ambientales, la estrecha vinculación entre las comunidades y las naciones y la gran cantidad de información disponible, que acarrea análisis de impactos presentes y futuros. Identificar las potenciales crisis externas, regionales y globales, requiere detectar tendencias, realizar análisis prospectivo y monitorear impactos y efectos ampliamente. Demanda, asimismo, de la capacidad de ver a través de la complejidad del mundo de hoy, identificar oportunidades y amenazas y desarrollar opciones y estrategias para aprovecharlas, enfrentarlas o mitigarlas, de preferencia en forma preventiva.

Es necesario contar con inteligencia territorial y competitiva, con una mirada abierta del mundo, ampliando los horizontes de evaluación, más allá de las tradicionales memorias, balances o informes anuales de gestión y sobre todo contar con equipos humanos capaces de realizar preguntas estratégicas y de análisis de escenarios, tales como: ¿Qué haremos en caso de x desastre? ¿Qué nueva oportunidad de emprendimiento, negocio o innovación turística podría crearse con estos riesgos, crisis, conflictos? Empresas, destinos, compañías y organizaciones públicas y privadas exitosas serán aquellas que reaccionen oportuna y estratégica y eficientemente frente a las crisis y las adversidades o riesgos, o aun más, sean capaces de avizorarlas y anticipar reacciones a ellas, antes que su competencia.

Riesgos, amenazas o crisis tienen una función tanto de probabilidad como de impacto, y ambas repercusiones pueden ser tremendamente subjetivas. En general las personas y los grupos humanos sobreestiman los riesgos cuando están familiarizados con ellos y subestiman los desconocidos, pues, obviamente, no saben cómo podrán afectarles. Por ende, la percepción y la gestión del riego y de las crisis, son afectados por la cultura, el grado de información y conocimiento disponible por los actores relevantes, el capital social desarrollado por las comunidades y/o la capacidad de resiliencia o tolerancia desarrollada ante los riegos, en su experiencia anterior.

Por otra parte, el World Economic Fourum (2005), identificó asimismo una serie de riesgos que amenazarán al planeta, en menor o mayor medida. Estas amenazas se agrupan en: económicas, geopolíticas, sociales y medioambientales. Aunque algunos de estos riesgos se ubican en una zona geográfica específica, todos tienen un impacto global. Véase, por ejemplo: inestabilidad política y militar en Medio Oriente, terrorismo y fundamentalismos radicales, crisis financieras, interrupción de los suministros de materias 
primas y energías estratégicas, cambio climático, fluctuaciones económicas y luchas entre las grandes potencias comerciales, pandemias, comercio de armas de destrucción masiva, migraciones y crisis humanitarias, desastres naturales (inundaciones, tormentas, aluviones, terremotos, erupciones), entre otras. De todos los riesgos mencionados, no existe ninguno que no afecte a los sistemas turísticos, los destinos y los países con industrias de viajes consolidadas o emergentes. Pero obviamente, la magnitud de los impactos puede variar. Tal es el caso, a modo de ejemplo, de la crisis financiera, que algunos países han enfrentado de una mejor manera que otros. Sin embargo, aunque no exista el mismo nivel de vida y poder adquisitivo para realizar viajes de larga o mediana distancia, todos se han visto afectados. Algunos subsistemas turísticos y subsectores de la industria son más vulnerables a una clase de amenaza u otra. Así, por ejemplo, las aerolíneas y el transporte aéreo en general, ha manifestado alta vulnerabilidad a las amenazas sociopolíticas y las situaciones de conflicto militar, mientras que el subsector de hoteles ha paralizado buena parte de los nuevos proyectos debido a la incertidumbre del crédito bancario y las inversiones de capital. Algunos destinos han sido más vulnerables a las amenazas de origen natural como aquellos situados en el Océano Índico o en el Cinturón de Fuego del Pacífico, a raíz de todos los desastres a los que se ven sometidos; terremotos, erupciones, huracanes, tsunamis, etc. (Sancho y Vélez, 2009).

La realidad se encarga de demostrar que los sistemas turísticos rara vez permanecen en equilibrio. Por el contrario, se ha llegado a la convicción de que "es el desequilibrio el que proporciona la estabilidad de estos", ante adversidades los agentes del sector y sus entornos cooperan en la lucha por la supervivencia. Se presume que los diversos elementos del sistema turístico tienen una importante comunicación entre sí y despliegan cierta organización, por lo que son capaces de dirigirse hacia la obtención de resultados o metas, obviamente más o menos exitosas, dependiendo de las estrategias, sus liderazgos y sus recursos y la experiencia acumulada.

Este modelo concibe la generación de sistemas complejos que están sujetos a perturbaciones provenientes del medio y los conducen a generar cambios y adaptaciones (Sancho y Vélez, 2009). Es importante, por ende, conocer qué cantidad de cambios será posible introducir en un sistema sin ponerlo en peligro, qué mecanismos desplegará para controlar o aprovechar las nuevas tendencias o una desviación de su trayectoria anterior y modificar la dirección hacia una situación potencialmente catastrófica.

La crisis y los desastres de origen natural difícilmente pueden ser evitados por los pueblos. Pueden considerarse eventos aleatorios, pero sí es posible prevenirlos en cierto grado, mitigarlos y reducir sus efectos adversos y sobre todo desarrollar estrategias ciudadanas e institucionales para preparar a la población frente a ellos. 
La protección y el entrenamiento ciudadano son las principales armas que puede ayudar en este tipo de crisis y en estos casos. Pero puede encontrarse un problema asociado a esta protección que es la corrupción que normalmente lleva asociada. Dentro de este ámbito hay que diferenciar entre los procesos de protección misma y los procesos compensatorios.

Los primeros sólo benefician a los destinos expuestos al riesgo y a aquel factor que lo origina, mientras que los segundos tienen igual beneficio para destinos expuestos y para otros no directamente expuestos a ese riesgo.

Para las crisis de origen antrópico y de carácter social, la identidad cultural que otorga a la población un sentido de identidad y permanencia constituye un factor clave que les permita afrontar y elaborar las influencias de culturas invasoras. Aquellos destinos que han hecho un baluarte del respeto y la exaltación de las culturas tradicionales han mostrado una mayor capacidad para recomponerse y renacer frente a numerosos conflictos de esta naturaleza. Esto no está reñido con la interculturalidad, que constituye una de las bases y de las principales consecuencias de la actividad turística. Es mas cuando un pueblo ha desarrollado más sus raíces es menos vulnerable a la acción de la interculturalidad. Las amenazas económicas son las que preocupan en estos momentos de crisis generalizada de la economía.

La OMT. (2009) ha elaborado un documento de respuesta a la crisis con un conjunto de medidas de los diferentes subsectores. Igualmente creó, en el año 2009, un comité para apoyar a los países a actuar con resiliencia después de las crisis generalizadas, entregando posibles respuestas para los diferentes subsectores.

\section{2.- Metodología.}

\section{1.- Como medir la resiliencia}

Para medir el grado de resiliencia de los destinos se pueden formular dos sistemas: un modelo basado en el análisis de un conjunto de variables claves y un modelo basado en el análisis comparativo de algunos factores entre varios destinos.

Este artículo ofrece una versión preliminar de la segunda forma de medición describiendo un modelo para su evaluación que se encuentra en periodo de prueba.

La fórmula creada se basa en utilizar un modelo shift-share para explicar las diferencias en intensidad de resiliencia en diferentes destinos, según la expresión: 


$$
R^{1}-R^{2}=\sum \bar{\beta}\left(F^{1}-F^{2}\right)+\sum\left(\beta^{1}-\beta^{2}\right) \bar{F}
$$

donde:

*R1 y R2 son las diferencias en intensidad de la resiliencia. $* * \beta$ son las diferencias de riesgo al que están sometidos los destinos ***F1 y $\mathrm{F} 2$ son los diferentes factores de riesgo.

El primer sumatorio de la expresión es el efecto en factores de riesgo del destino, y el segundo sumatorio es el efecto en riesgos de los destinos.

Esta metodología ayuda a los municipios a aprender de la experiencia de los otros destinos, estimar el impacto que la amenaza ha generado sobre los demás, y reconocer un incremento en bienestar y mejora de calidad. Pero para ello hay que tener claro cuales son los factores y variables que facilitan o fortalecen la resiliencia.

Basándose en esta fórmula desarrollada para cuantificar la resiliencia se presenta aquí una primera aproximación a esta valoración, centrada en el estudio de dos destinos turísticos del sur de Chile (Valdivia-Corral y cuenca del lago Ranco, ambos de la región de Los Ríos a 800 kms. aproximadamente de Santiago de Chile, la capital del país).

Se ha construido una encuesta para los gestores locales de estos dos destinos, valorando los tres factores que influyen sobre la resiliencia: la autoridad real de los recursos, las capacidades de los destinos de gestionar los subsistemas de manera competente, distribuir equitativamente los ingresos generados por esta actividad turística y las conexiones que tenga esta actividad con el resto de toda la economía (Ecoespaña, 2009). Sehanvaloradolasmedias paracadagrupo de factoresposicionandoacadaunodelos destinos en función de estas diferencias. Con ellas se han obtenido los siguientes resultados en una primeraaproximación, considerandoqueestetrabajoespartedeunainvestigaciónmásamplia.

\section{2.- Ámbito territorial.}

El estudio se ha aplicado en dos destinos de la región de los Ríos del sur de Chile:

a) Destino Valdivia-Corral

Este destino está compuesto por las comunas de Valdivia y Corral y posee la estructura receptiva y de hospitalidad más importante de la región de Los Ríos, actuando como eje vertebrador y centro receptor y distribuidor. Se ubica en un sistema hidrográfico formado por tres cuencas: el río Calle-Calle, que se 
caracteriza por tenerun caudal abundantey relativamente uniforme durante todo el año; el río Cruces, declarado Santuario de la Naturaleza en su curso inferior y el río Tornagaleones, que recibe por la ribera oriental dos ríos de curso paralelo y de poco desarrollo: Futa y Angachilla. La comuna de Valdivia tiene una población de alrededor de 150.000 habitantes, con una población joven que no muestra signos aún de iniciar un proceso de envejecimiento.

Los principales accesos a este destino son:

Vía terrestre: desde el norte, la autopista panamericana que se bifurca, al llegar a San José de la Mariquina, hacia el suroeste en un tramo de $47 \mathrm{kms}$. hasta llegar a la ciudad de Valdivia, Desde el sur la misma vía, se bifurca en Paillaco hacia el noroeste, en un tramo de $48 \mathrm{kms}$. hasta llegar a la ciudad de Valdivia.

Vía aérea: El aeropuerto Pichoy dista $32 \mathrm{kms}$. al noreste de Valdivia Actualmente existe una disociación entre la oferta y la demanda en este destino, con una sobreoferta desde el punto de vista de la demanda actual. Por lo tanto, podemos decir que este destino se encuentra desarrollado desde el punto de vista de la oferta, no así de la demanda. Si bien ésta no ha disminuido, su estancamiento ha sido factor negativo para el desarrollo turístico del destino, así como la tendencia a la baja que se observa en el poder adquisitivo de los visitantes de verano (Figueroa et. al, 2018)

Respecto a su potencial turístico este destino se caracteriza por contar con el centro urbano más importante de la región, que es a la vez su capital cultural, artística, de negocios, educacional, científica, lo que la convierte en el principal centro receptor del flujo de viajeros que se movilizan durante el año por esa amplia gama de motivaciones.

De igual forma, en verano se convierte en una atractiva ciudad fluvial, de hermoso diseño, única por su ubicación a la orilla del río navegable más importante del país, por sus bellos paisajes, su conexión al Océano Pacífico, su tradicional gastronomía, sus festividades nacionalmente conocidas y por ser el punto de partida de excursiones fluviales río arriba y hacia la desembocadura de la cuenca donde se encuentra la red de fortificaciones y monumentos históricos legado de la colonización española y declarados patrimonio nacional de importancia internacional (Szmulewicz et. al. 2010).

El destino Valdivia - Corral cuenta con toda la gama de servicios urbanos necesarios para visitantes y con equipamiento turístico diverso, amplio, apto para todos los segmentos y que abarca hotelería, restauración, transporte, esparcimiento, e instalaciones para actividades culturales, recreativas, deportivas, etc. Una de las características que distingue a Valdivia es su completa oferta de servicios de esparcimiento nocturno, en gran parte derivado de su condición de ciudad universitaria y de eventos. De igual 
forma, otro de los sellos de la oferta de servicios turísticos es la amplia variedad de embarcaciones que realizan tours y transporte a través de su red fluvial. Corral por su parte destaca como puerto receptor de las excursiones fluviales y por contar con una creciente oferta de actividades de turismo rural y de naturaleza en su entorno. El destino Valdivia- Corral se encuentra en un estado de madurez, como área turística, en la cota superior de la curva, presentando en los últimos años algunos signos preocupantes de estabilidad en el número de visitantes y de tendencia hacia segmentos de menor poder adquisitivo, con posibilidades de girar hacia una declinación o una reconversión. En este destino se pueden identificar diversas organizaciones del empresariado turístico y la presencia de múltiples instituciones ligadas al sector, destacando además la existencia de varias escuelas de formación de personal técnico para esta industria y especialmente de formación profesional en la escuela de Turismo de la Universidad Austral de chile, cuyo impacto sobre el desarrollo del sector ha sido notable a lo largo de sus más de 40 años de existencia. A pesar de contar con esta gran presencia de organizaciones ligadas al sector, lo que debería ser una fortaleza se convierte en una debilidad, cuando existe atomización y su capital social, siendo muy notable ofrece hoy signos claros de problemas de desagregación y falta de coordinación y de trabajo conjunto entre todas las organizaciones (Szmulewicz et. al. 2010).

b) Cuenca del Lago Ranco de la Región de los Ríos, Chile. Comprende la cuenca del lago mencionado y sus áreas circundantes, y dentro de él se encuentra todo el territorio de las comunas de Futrono y Lago Ranco y gran parte de las comunas de la Unión (capital provincial), Río Bueno y Paillaco. El uso del suelo y la vegetación que presenta una mayor representación en la cuenca es el bosque nativo, que le otorga alta biodiversidad. Tienen representatividad en la cuenca 3 áreas protegidas del Estado. Además, de importantes áreas de protección privadas.

La base productiva de la Cuenca del Lago Ranco gira en torno a agricultura, ganadería, apicultura, silvicultura, industria manufacturera (madera y agricultura), artesanías, comercio y turismo. En los últimos años han surgido importantes proyectos de conservación de la biodiversidad y de generación hidroeléctrica.

El principal recurso turístico de la zona lo constituye el lago Ranco ("aguas tormentosas" en lengua mapuche), con $442 \mathrm{~km} 2$ de superficie y $199 \mathrm{~m}$ de profundidad. Posee alrededor de 13 islas, ocupadas mayoritariamente por casas de veraneo y comunidades de pueblos ancestrales. Entre ellas, destaca isla Huapi.

Los otros atractivos naturales más importantes corresponden a lagos y lagunas, el ecosistema Selva Valdiviana, que se caracteriza por una cubierta vegetal tupida continua, con bosque de varios estratos, sus ríos y saltos, playas e islas y sus termas. 
La oferta turística se desarrolla en torno al turismo vacacional y residencial de sol y playa. No obstante, cuenta con recursos turísticos que le permiten avanzar hacia la estructuración de oferta turística en las modalidades de turismo de intereses especiales (pesca recreativa, agroturismo, ecoturismo, etnoturismo, cinegético, entre otras) (Figueroa et. al, 2018) Entre los atractivos culturales del destino, destacan eventos programados, museos, circuitos patrimoniales arquitectónicos y las visitas a comunidades indígenas complementadas con artesanías y actividades productivas tradicionales. En la comuna de Lago Ranco existen 27 comunidades indígenas y 6 asociaciones indígenas reconocidas, mientras que, en Futrono, existen 12 comunidades y 2 asociaciones.

\section{3.- Desarrollo}

Los resultados preliminares de la valoración hecha en los dos destinos ponen de manifiesto la importancia que tienen las conexiones para mantener un nivel significativo de resiliencia. Cuando estas conexiones son predominantemente de carácter local y regional, se establece un nivel de gobernanza importante, cercano a la población y se presentan condiciones favorables para liberar el potencial económico de los diferentes subsistemas turísticos, de forma que realmente alcance a prácticamente todos los actores del destino y desarrolle capacidades de anticipación y adaptación continua a las tendencias profundas y de largo plazo que puedan interferir con la evolución turística. No obstante, el despliegue de esas capacidades depende, en forma significativa, de la capacidad de inteligencia territorial y económica que posean los destinos para monitorear las tendencias y los cambios en los mercados y en aspectos económicos y socio culturales en general. Eso es lo que se observa débil en ambos destinos, pero, obviamente, más en un destino rural, de baja población y débil contextura profesional como la cuenca del lago Ranco, que, en un destino urbano, universitario y cultural, como Valdivia.

Para las crisis y eventos imposibles de predecir, incluso con los métodos más sofisticados, la comunicación y la articulación comunitaria y de las instituciones expertas, puede ayudar a vislumbrar y poner en marcha formas para prevenir, reducir y mitigar los impactos negativos, sobre todo para los grupos y actividades más vulnerables. La vulnerabilidad obedece primordialmente al riesgo, a la incertidumbre y a la falta de programas actualizados de prevención y de seguridad. Esto obliga, por tanto, a que el nivel de comunicación y organización al interior y entre los destinos de características similares sea muy valorable.

La organización, liderazgo y la cohesión social en estos destinos, y un modelo de gobernanza participativa, resultan ser factores claves, de carácter transversal, que ayudan a crear resiliencia ante fenómenos que pudieran provocar desastres, ya que al incrementarse reducen significativamente la vulnerabilidad respecto a las amenazas. 
Los problemas ambientales en la Región de Los Ríos, donde se encuentran Valdivia-Corral y la cuenca del lago Ranco, se han acentuado por la puesta en marcha de un modelo de desarrollo que ha profundizado las contradicciones en la relación turismo-medioambiente. Dentro de los factores que potencian el riesgo se pueden mencionar la debilidad institucional, la ineficiencia de las políticas públicas, legislación y normas inadecuadas o no aplicadas, sistemas deficientes de seguimiento, poca a nula información, observación, vigilancia y alerta temprana, escasez de capital financiero, físico, humano y social, entre otros factores.

En ninguna de las dos zonas estudiadas existe una planificación precisa y una activa participación establecida, de la población local, en los escasos programas de prevención y recuperación ante desastres y situaciones de riegos. Tampoco se cuenta con planes de ordenamiento territorial que aseguren el uso adecuado de los espacios, de modo de minimizar los riesgos naturales, posibilitar el uso sostenible de los recursos naturales, garantizar inversión en infraestructura apropiada a las adversidades de los territorios, de calidad y compatible con el desarrollo de un modelo de turismo asociado a la naturaleza y a la preservación de las tradiciones culturales regionales. Las actividades productivas locales y el desarrollo urbano se han establecido sin considerar la existencia de áreas expuestas a amenazas naturales. Respecto a las infraestructuras no se manifiesta una mejora de éstas a causa del turismo para los poblados de la cuenca del Ranco, como sí es reconocido su aporte en Valdivia. Se debe recalcar que Valdivia como ciudad capital de la región posee más alternativas de desarrollo que Futrono, Paillaco, Río Bueno, La Unión o Lago Ranco (las ciudades de la cuenca lacustre) lo que la hace tener una ventaja comparativa frente a los demás destinos. Los actores locales de la cuenca del Ranco sí que consideran que los caminos y la calidad del servicio público ha mejorado, a diferencia de la opinión de los actores de Valdivia que no lo reconocen. Esta diferencia de opiniones podría deberse a la diferencia de estándares de calidad con los que se emite el juicio, puesto que la más mínima mejora en el destino cuenca del Ranco será percibida de manera inmediata ya que es un destino más pequeño a diferencia de Valdivia donde sólo será percibida por aquellos que utilizan servicios públicos.

De igual modo las encuestas reflejan desconocimiento de normas, protocolos y procedimientos de prevención y gestión ambiental. A lo anterior se suma la escasa preparación de la población para reconocer, valorar y enfrentar las amenazas y situaciones de desastres, con mentalidad preventiva o mitigadora.

Del mismo modo, el turismo contribuye de menor grado el fomento de actividades culturales. Lo anterior se podría fundamentar a través del surgimiento de actividades culturales que están siendo incluidas en menor volumen en los programas de actividades turísticas. El Turismo cultural o la incorporación de actividades culturales lentamente comienza a ser parte de la oferta turística, pero la descoordinación de instituciones 
hace difícil la tarea de complementar las actividades ofrecidas en turismo con aquellas de ámbito cultural. En el destino lago Ranco, se observa que el fomento que realiza el turismo sobre las actividades culturales del destino es mayor que en Valdivia. Esto puede ser debido a que son destinos diferentes, de tamaños distintos, uno de ellos con vías de comunicación más directas, donde la población local está más cerca y por ende se informa con mayor rapidez de actividades que puedan ser incluidas en las ofrecidas por el turismo; que son, además, el motor de movimiento de turistas para el destino.

Considerando los datos desde una mirada global, las opiniones indican que los recursos como: agua, aire, ruido, y planta de tratamiento de aguas no se ven tan afectados por la llegada de turistas. Los destinos en estudio se encuentran ambos ubicados en zonas ricas en los tipos de recursos mencionados, por estar emplazados en orilla de ríos y lagos y la ventilación característica de ellos hace mermar el efecto de la contaminación de aire y ruido. Respecto al tratamiento de aguas servidas, los destinos han invertido recientemente en ampliación de sus plantas, lo que transmite seguridad respecto a este tema. Por el contrario, los niveles de tránsito de peatones, congestión vehicular y limpieza de las ciudades se ven afectadas de manera negativa con la afluencia de turistas. Los pocos vínculos entre la planificación del desarrollo, el uso del territorio, las condiciones naturales de la región, sus recursos y la gestión del riesgo, conforman un panorama poco halagador que refuerza el diagnóstico de importantes carencias de capacidades para enfrentar adversidades y riesgos. La situación se complica por la carencia de memoria histórica acerca de las causas y consecuencias de los desastres y la ausencia de opciones suficientes para los riesgos que los hace a su vez más vulnerables.

\section{4.- Conclusiones}

Muchos estudios del fenómeno de urbanización reconocen que el rápido crecimiento turístico en el mundo en desarrollo es inevitable, pero no aceptan las nefastas consecuencias que acarrea. Se asume que los gobiernos municipales con buena capacidad de gestión pueden absorber un gran aumento turístico sin disminuir el bienestar humano ni la calidad del medio ambiente. La clave reside en el compromiso de introducir y sostener políticas que, entre otras cosas, fomenten el capital social, mantengan la infraestructura, aumenten la productividad de la fuerza laboral, combatan los delitos contra los recursos naturales y la economía en este proceso como es el urbanismo ilegal. Para ello, es interesante potenciar la gobernanza para una mejor comprensión del funcionamiento de los destinos turísticos, y una gestión más competitiva y sustentable.

El gobierno eficiente en las ciudades y municipios es necesario para superar las presiones impuestas por el crecimiento demográfico. Aunque las prácticas de buen gobierno municipal son de introducción reciente y aún no se han ejecutado en su 
totalidad en ninguna parte, incluyen aspectos como la participación de los actores no gubernamentales (comunidades, grupos cívicos, contratistas privados) en la atención de las necesidades básicas; la descentralización del poder de adopción de decisiones y del control de los recursos municipales a grupos locales autóctonos; y una respuesta más amplia de los gobiernos municipales a las necesidades locales, mediante mayor responsabilidad por sus obras y más transparencia en materia de financiación.

Por la naturaleza misma del producto / experiencia turística existe una costumbre de formas de colaboración público - privada que se materializa en la transversalidad de la actividad turística, por las externalidades que genera el desarrollo turístico. Es importante generar redes interterritoriales que favorezcan las acciones conjuntas.

La gestión ambiental se ha impulsado como una estrategia para reducir la vulnerabilidad, creando políticas que faciliten la conservación y manejo de los recursos naturales y prácticas específicas para fomentar el desarrollo socioeconómico en los dos destinos. Al realizar un análisis comparativo entre destinos, se puede observar que existen opiniones similares respecto a que los recursos de consumo de agua y capacidad adecuada de planta de tratamiento de aguas servidas no se ven tan afectados por la llegada de turistas. Sin embargo, es Valdivia quien considera menor el impacto de los turistas en estos ámbitos, esto puede deberse a que como ciudad capital con un mayor desarrollo en muchos sentidos se siente más preparada para la llegada de los turistas. Por el contrario, las ciudades de la cuenca del Ranco al ser más pequeñas notan de inmediato el aumento de consumo de recursos, pues están menos preparadas que Valdivia. Asimismo, ambos destinos coinciden en argumentar de manera similar que la llegada de turistas trae consigo un aumento de la congestión de personas que dificulta el caminar.

Se argumenta también que no se cuenta con la debida protección de los recursos naturales. Además, estas opiniones se han visto reforzados por situaciones ambientales (estudios de impacto ambiental de centrales hidroeléctricas) que ha generado un movimiento masivo en contra de acciones que atenten a la conservación de las áreas.

Las principales diferencias entre destinos se encuentran en que Valdivia percibe efectos negativos en su calidad de aire y la emisión de ruidos provocados por la afluencia de turistas, a diferencia de la cuenca del Ranco que se manifiesta de manera contraria, donde a raíz de los visitantes no se generan estos efectos. Lo anterior podría deberse a que los turistas que visitan Valdivia se que dan en la ciudad, aloja, comen y se entretienen en la ciudad, a diferencia del destino lago Ranco, donde la mayoría de los visitantes desarrollan gran parte de sus actividades en las zonas rurales, que es donde se ubica la gran cantidad de oferta de alojamiento. 
Respecto a la limpieza, los destinos indican diferencias de opinión. Para Valdivia, el turismo no ha contribuido al deterioro del aseo, por el contrario, se podría inferir que bien ha producido un mayor cuidado por parte de las autoridades pertinentes. La opinión en la cuenca del Ranco es opuesta, considerando que, debido al turismo, los niveles de limpieza han empeorado en las ciudades. El empeoramiento de la limpieza podría estar vinculado con la necesidad de contar con una mayor cantidad de personal de aseo, que refuerce este ámbito. Si con el aumento de turistas se tiene al mismo personal, las calles estarán aparentemente más sucias, la frecuencia de recolección de basura será inferior a la necesaria.

La integración de la gestión del riesgo en los procesos de planificación municipal y regional depende de la voluntad, actitud de diálogo, negociación y fortalecimiento de alianzas entre gobiernos locales, organizaciones, líderes comunitarios, instituciones del Estado, etc.

Esnecesarioreducirlavulnerabilidadpormediodeunprogramadeinversionesymejorasenla capacidaddeorganizacióndelassociedades, concluyendoenmejorcalidaddevida,reduciendo el riesgo y la afectación psicológica de los pobladores, incorporando medidas de mitigación y prevención, ya que la prevención minimiza el daño y promueve un ambiente estable.

\section{5.- Bibliografía}

Banchini s. (2005). Resiliencia de los destinos turísticos frente a la velocidad de actuación de las nuevas Aerolíneas de bajos coste. Instituto de Arquitectura Avanzada de Cataluña.

Blanco, R. (2009). Presentación agenda 21 del turismo sostenible para entidades locales. Blanco, R. (2008). Presentación diseño de un método para el seguimiento de la sostenibilidad del turismo en destinos municipales de la red de reservas de la biosfera: sistema de indicadores. Subdirección General de Calidad e Innovación Turística. Cabrer, B. et. al. (2010) El turismo sostenible, un reto para los destinos. Estudio comparativo para destinos emergentes de la Región de los Ríos en el sur del Chile. Aportes y Tranferencias, Año 14, V. 2: 48-60.

CDMB (2009):. La reducción del riesgo y su gestión. Corporación Autónoma Regional para la Defensa de la Meseta de Bucaramanga http://www.cdmb.gov.co Declaración de Santa Cruz +10 (2008). Primera Reunión Interamericana de Ministros y Altas Autoridades De Desarrollo Sostenible. Organización De Los Estados Americanos. Consejo Interamericano para el Desarrollo Integral (CIDI). Santa Cruz de la Sierra, Bolivia.

ECOESPAÑA (2009): Las raíces de la resiliencia - Aumentar la riqueza de los pobres. Fundación Biodiversidad. Recursos Mundiales. Madrid. 
García, G. (2005). Medición de la capacidad de carga de la población local y los turistas en un destino turístico. Tesis Doctoral. Universidad de Valencia Hamel, G. y Välikangas, L. (2003). En busca de la resiliencia. Harvard Business Review, ISSN 0717-9952, Vol. 81, Nº. 9, 340-52.

Henderson E. (2006). LA resiliencia en el mundo de hoy. Ed. Gedisa, Buenos Aires Internacional Monetary Fund (1995): Resilience and Growt Through sustains adjustment. Washington. USA.

Jiménez Herrero L.M. (2002). La sostenibilidad como proceso de equilibrio dinámico y adaptación al cambio. ICE. 65- 84.

Luthar S.S: (2003): Resilience and vulnerability. Ed Cambridge Prees .USA. Melillo A. y Suarez E:N. (2001): Resiliencia, descubriendo las propias fortalezas. ED. Paidós. Buenos Aires.

Ministerio de Medio Ambiente Y Medio Rural Marino (2009): Perfil ambiental de España: Informe basado en indicadores. Madrid.

Organización Mundial de Turismo (OMT.) (2005). Indicadores de desarrollo sostenible para los destinos turísticos. Guía práctica. Madrid, España.

Organización Mundial de Turismo (OMT.) (2009). Informe sobre Resiliencia. Comité para la resiliencia. Madrid.

Osorio, M. (2005). La teoría de sistemas aplicada al turismo. Pensando turismo. http:// pensandoturismo.com

Secretaría General de Medio Ambiente (2003). Sistema español de indicadores ambientales de turismo. Dirección General de calidad y Evaluación Ambiental Ministerio de Medio Ambiente (Editor).

SanchO, A., García, G. y Rozo, E. (2007). Comparativa de Indicadores de Sostenibilidad para destinos Desarrollados, en Desarrollo y con poblaciones Vulnerables. En Annals of Tourism Research en Español. Vol 9 No 1: 150 -176. Sancho A. Velez, Y. (2009). La resiliencia como metodología para enfrentarse a las crisis del sector turístico. Fundación Ramón Areces. AECIT Szmulewicz, P. Gutiérrez, C. et. al. (2010). Modelo de Innovación en Productos Turísticos. Edita: UACh.-U de Queensland, Australia. Valdivia, Chile: 99 pp. (Registro Propiedad Intelectual: 195090 ISBN: 978956-7105-53-3)

WORLD ECONOMIC FORUM. (2005). Global Risk to the Business Envoronment. With the collaboration of Merrill Lynch. 